\title{
How malnutrition is detected and managed in patients with spinal cord injuries (SCI): results from a UK multi-centre study
}

\author{
S. S. Wong ${ }^{1,2}$, F. Derry ${ }^{1}$, A. Jamous ${ }^{1}$, G. Grimble ${ }^{2}$ and A. Forbes ${ }^{2}$ \\ ${ }^{1}$ National Spinal Injuries Centre, Stoke Mandeville Hospital, Aylesbury and ${ }^{2}$ Centre for Gastroenterology and Clinical \\ Nutrition, University College London Hospitals, London, UK
}

\begin{abstract}
Malnutrition has severe adverse effects on health outcomes and healthcare expenditure ${ }^{(1)}$. However, recognition and treatment of malnutrition (under/overnutrition) in patients with spinal cord injuries (SCI) in SCI centres (SCIC) are not often a priority in clinical practice $^{(2)}$ and data on the prevalence of malnutrition in SCI patients are limited. The aims of the study were to (1) investigate how nutritional risk of patients is determined across all twelve SCIC in the UK and Ireland; (2) establish the prevalence of malnutrition using a generic nutrition screening tool (NST), Malnutrition Universal Screening Tool (MUST) ${ }^{(3)}$ and BMI. After obtaining ethics approval, 11/12 SCIC participated in part 1 of the study. Eight used NSTs, three had no dedicated staff member for nutritional care, and nine SCIC reported that they did not have a clinical nutrition team. Four SCIC, contributing $49 \%$ of the SCI beds in the UK, participated in part 2 of the study from July 2009 to March 2010. One hundred and fifty patients (aged 18-88, median: 16.9, 30.7\% female) were studied after obtaining written informed consent. The Malnutrition MUST score, baseline clinical data, anthropometric measurements and blood biochemistry were assessed within $96 \mathrm{~h}$ of admission. Differences between independent groups were assessed with the Mann-Whitney test. The risk of undernutrition was defined from a MUST score $\geq 1$ and overweight was defined from BMI $\geq 25$ and $\geq 30$ as obese. The present study found that $45.9 \%$ of patients were overweight and $15.3 \%$ were obese; the prevalence of undernutrition risk was $44.3 \%$. Patients who were at risk of undernutrition were found to have significantly reduced total protein, albumin, $\mathrm{Mg}$, creatinine, Hb, BMI, appetite and significantly higher C-reactive protein, and received more prescribed medications. The process of nutrition risk screening varied between SCIC and may lead to under-detection and under-management. The very high percentage of SCI patients who are at risk of malnutrition is worrying, given the adverse consequences for short- and long-term health and well-being. Strategies for systematic screening and treatment of malnutrition in SCI patients need to be refined and implemented. Further research on the best combinations of simple clinical indices relevant to patients with SCI is warranted.
\end{abstract}

The authors would like to thank Wendy Nicholls, Stoke Mandeville Hospital, Anthony Twist, Robert Jones and Agnes Hunt Orthopaedic and District Hospital, Philippa Bearne, Salisbury District Hospital, Dr. Angela Gall and Judith Susser from Royal National Orthopaedic Hospital for data collection and Abbott Nutrition for the financial support. UCL Staff receive support from the CBRC funding awarded to UCL and its partner Trust by NIHR.

1. Brotherton A, Simmonds N \& Stroud M (2010) Malnutrition Matters: Meeting Quality Standards in Nutritional Care. BAPEN, Redditch.

2. Wong SS, Derry F, Grimbel G et al. (2011) Does knowledge of nutrition is a potential barrier to implement better nutritional care? An audit to assess knowledge about malnutrition among staff working in the UK SCI centres. Proc Nutr Soc 70 (In the Press).

3. Elia M (2003) Screening for Malnutrition: A multidisciplinary Responsibility. Development and Use of the Malnutrition Universal Screening Tool (MUST) for adults. BAPEN, Redditch. 